\title{
Probability and Rational Choice
}

\author{
DAVID BotTING
}

\begin{abstract}
In this paper I will discuss the rationality of reasoning about the future. There are two things that we might like to know about the future: which hypotheses are true and what will happen next. To put it in philosophical language, I aim to show that there are methods by which inferring to a generalization (selecting a hypothesis) and inferring to the next instance (singular predictive inference) can be shown to be normative and the method itself shown to be rational, where this is due in part to being based on evidence (although not in the same way) and in part on a prior rational choice. I will also argue that these two inferences have been confused, being distinct not only conceptually (as nobody disputes) but also in their results (the value given to the probability of the hypothesis being not in general that given to the next instance) and that methods that are adequate for one are not by themselves adequate for the other. A number of debates over method founder on this confusion and do not show what the debaters think they show.
\end{abstract}

Keywords: Probability; rational choice; confirmation of hypotheses; singular predictive inference; inductivism; falsificationism.

\section{Two Choices}

The future is unknown. What is worse, it is not directly knowable - we cannot see into the future. If the future is knowable at all, if we are to have rational expectations about what it holds, then it can only be indirectly by drawing inferences from what we do know and from the evidence that we do have. These evidence statements are about the past and the present. So, any inference from the evidence to the future must be, by definition, ampliative and deductively invalid. How, then, does this evidence ground the rationality of our beliefs about the future?

The traditional answer has been induction. The past is a guide to the future because the future resembles the past. The most general answer we can give to the question of what the future holds is "more of the same", the evidence of the past being taken as a fair sample of the whole of time, including the future. Whether we are rational in believing this has exercised the greatest minds for centuries. If by 'rational' we mean justified either deductively or inductively, then Hume argues persuasively that no ampliative inference is justified; if it could, then it would not be ampliative. Inductivists nonetheless insist that we can attach probabilities to hypotheses by inferring from the sample to the population, which being so makes it rational to believe hypotheses that are very likely.

Principia 18(1): 1-24 (2014).

Published by NEL — Epistemology and Logic Research Group, Federal University of Santa Catarina (UFSC), Brazil. 
I will refer to this criterion of selection in the rest of the paper as the probability of the hypothesis, but this terminology is somewhat ambiguous. The probability referred to here is the probability that is part of the content of the hypothesis, e.g., the $\frac{8}{10}$ in "Eight out of ten cats prefer Bird-U-Like" and not how likely this statement is to be true. These are sometimes called the empirical probability (the probability of the hypothesis) and epistemic probability respectively. This is a distinction of the utmost importance, for I will show later that we can learn from evidence even if the evidence does not alter the empirical probability because it does alter the epistemic probability, and that this does not change which hypothesis is rational to select but does change the value we should give for the next instance.

The alternative answer is falsificationism. Inductive reasoning not only fails to show that its claims logically follow from the evidence for them but even fails to show that they probably follow, says Hume; yes, agrees Popper, therefore it can never be rational to believe in a hypothesis, and the inductive inference is no kind of inference at all. The whole idea of attaching probabilities to hypotheses and saying one is more likely to be true than another is misconceived and does not take Hume seriously enough. Nevertheless, Popper allows that we can make rational choices based on evidence about which hypotheses to select. Here there are two criteria for selection, corroboration and empirical content. Of two conjectures that are equally well corroborated (have passed critical tests aimed at their falsification) we should choose that with the highest empirical content and of two conjectures equal in empirical content we should choose the best corroborated. The evidence now appears in the form of corroboration statements about how well hypotheses have survived attempts to falsify them. Popper denies that these statements say anything at all about the future or are a guide to the future, yet he does seem to say that it is more rational to choose a better corroborated hypothesis over a less well corroborated hypothesis despite the fact that one is not more likely to be true than the other. The choice is a methodological statement rather than a truth-claim.

The choice between inductivism and falsificationism will be discussed in some detail later. But to give the reader a clue, there is a perfectly understandable sense in which we use the term 'rational' as applied to choice rather than belief that does not need to be justified. It is often rational to choose to act on the basis of a hypothesis one does not believe; for instance, if you need to achieve $\mathrm{Z}$, and $\mathrm{Z}$ is only achievable under circumstances $C$, then it is rational to act as if $C$ were true even if you believe it to be false. This is only a practical attitude towards a proposition. ${ }^{1}$

When the aim is 'theoretical', that is to say, we wish to select those hypotheses we should build our explanatory picture of the world from, the rationality of this choice depends for the inductivist on the probability of the hypothesis and for the falsificationist on the degree of corroboration and empirical content. I will argue that both inductivist and falsificationist rely on the concept of rational choice, and 
rely on it in a way that is (perhaps surprisingly) similar; the arguments of both sides, although they seem to be in competition, rely on almost identical claims about rational choice and seem to stand or fall together. Even if Popper is right to say that it is irrational to believe a hypothesis, the inductivist may still be rational to choose the most likely provided that there is a most likely and Popper is wrong about attaching probabilities to hypotheses. The later discussion is designed to show the parallelism rather than to decide who has the better answer to the problem of rationally selecting a hypothesis (although I tentatively side with inductivism).

It is not the choice between inductivism and falsificationism with which this paper is primarily concerned. Rather it is the choice between the 'theoretical' and the 'practical'. Not every expectation about the future has the theoretical aim of understanding the universe. Sometimes we just want to know what will happen next. This is often called inference to the next instance or the singular predictive inference. For the inductivist this is an inference from the sample to the next instance, and what I want to show is that this inference does not depend solely on the probability of the hypothesis, although it does depend only on the evidence. In the next section we will discuss, under the name of 'practical prediction', whether the falsificationist has a rational method for making this kind of inference.

The contrast is revealed in two kinds of questions, exemplified by "What is the probability that all the balls in the bag are white?" and "What is the probability that the next ball that I draw will be white?" Often these are taken to have the same answer, ${ }^{2}$ and can be taken so even if we eschew talk of probability, since on the face of it the outcome we should rationally expect for the next instance should be that which is predicted by the hypothesis we should rationally select. However, I believe it to be an error to think that the probability or degree of corroboration of a hypothesis can unproblematically be translated into a measure of rational choice for use in games such as gambling. Thus, I would propose the following Probability-Choice Disconnection Thesis: when we are choosing with other than theoretical aims, more factors affect what it is rational to choose than the probability of the hypothesis. ${ }^{3}$

We can see this in the limit case where, because there have been no trials and hence the probability of the hypothesis cannot be estimated, one can still choose rationally by the principles of classical (not frequentist) probability if one knows the alternatives between which one can choose. Here is a basic scenario in classical probability to illustrate: suppose that there are two bags, one with twenty white balls and one with nineteen white balls and one black ball, but you don't know which is which. Thus you are choosing a random ball from a random bag. What is the probability that the ball you draw will be white? Obviously we now have two factors. The probability that you draw a white ball will be the probability that you choose the bag with all white balls (0.5) multiplied by the probability that you pick a white ball from that bag (1.0) plus the probability that you choose the other bag (0.5) 
multiplied by the probability that you pick a white ball from that bag (0.95). The probability that the ball you draw will be white is then $((0.5 \times 1.0)+(0.5 \times 0.95))=$ 0.975. One factor is the probability itself, based on the evidence of what is in the bag, and the other is the weight of evidence, here decided a prioristically by equal division over the logical possibilities (i.e., 1.0 divided by the number of bags). In the limit case the evidence of what is in the bag is not something that we have learned from drawing balls from the bag, which is to say that the evidence statements are not probability (i.e., frequency) statements. It would, then, be slightly misleading to think of 0.95 and 1.0 as the probabilities of their respective hypotheses. Until a ball has been drawn the probability of the hypothesis simply cannot be estimated. However, it seems clear that one can rationally infer to the next instance. Then, when the first ball is drawn the probability of the hypothesis is 1.0 if the ball is white and 0.0 if it is not. One might be tempted to think that the probability should be estimated as less on the grounds that we still do not know from which bag the ball is being drawn, but this mistakes the probability of the hypothesis with the weight of evidence - you may be less certain that the hypothesis has that probability (have a lower epistemic probability), but this is not the same as believing it to have a lower (empirical) probability. ${ }^{4}$ The latter choice would be an irrational one.

The Probability-Choice Disconnection Thesis has the immediate consequence that whatever rational method is sufficient for estimating the probability or degree of corroboration of the hypotheses (I will argue later that the Straight Rule could be such a method for the former) is insufficient to solve the problem of the singular predictive inference. When the issue is over the truth of hypotheses, ('theoretical') choice and belief can come together, but as we have seen illustrated above the same may not be true when the issue is over the ('practical') choice of how best to spread one's bets on the next instance. In later sections it will transpire that this is because the singular predictive inference includes, as Carnap describes in his $\lambda$-system, a logical factor that functions as part of what determines betting quotients for instances but operates differently (i.e., on the epistemic probability) with regard to the truth or probabilities of hypotheses.

In a certain sense, then, the choice between theoretical and practical aims is the choice between classical and frequency interpretations of probability and between correspondingly different interpretations of modal statements. I said above that it was in some ways misleading to say of the report of a complete enumeration such as "All the balls in the bag are white" as hypotheses whose probabilities for any particular trials could then be derived a prioristically. I will now show that it has to be interpreted as a categorical statement, and that this is different from a probability or frequency statement.

A statement like "All Xs are Y" does not mean the same when uttered in the usual way as a categorical statement as it does when uttered as a probability statement. As 
a categorical statement it can be considered extensionally as reducible to a conjunction of singular statements; if the number of conjoined statements is finite then the truth of the categorical statement is established conclusively when each conjunct is established conclusively, that is to say, on a completed enumeration, but if the number is infinite then the truth of the categorical statement can never be established conclusively and the statement is - according to several well-known accounts of meaning including Reichenbach's - strictly meaningless. Whatever meaning it has, it has it in virtue of saying something distributively about each member of a set, as reflected when we give its logical form as including a universal quantifier, viz., $\forall x . X(x) \supset Y(x)$.

When uttered as a probability (frequency) statement, "All Xs are Y" means that the relative frequency of Xs in the set of Ys converges in the long run towards a limit of 1.0. The probability statement is not reducible to an infinite set but is a singular statement about an infinite set that does not, in general, say anything at all about any particular member of the set and is meaningless if such a set is logically impossible. This can be symbolized as $\forall x . X(x) \supset^{1.0} Y(x) .{ }^{5}$ Note that here, oddly perhaps, "all" does not mean "without exception", since the frequency ratio of Xs to Ys may converge on 1.0 even if there are Xs that are not Ys, provided that the number of Xs that are not Ys is finite or their frequency of occurrence is a decreasing function of the size of the sample; this means that the categorical statement can be false, and conclusively established as false, when the probability statement is true. This scenario is rather artificial, however, and it is questionable whether the frequency series that would be formed in these artificial cases are of the correct kind that can properly be said to have probabilities since the events do not seem indefinitely repeatable or indifferent to their ordering. The general rule that probability statements can never be conclusively established or falsified applies even for the extreme values of 1.0 and 0.0 ; the categorical statement can be true and established conclusively as true (by completing the enumeration) without the probability statement being also conclusively established as true, and we have already seen that establishing it conclusively as false does not establish conclusively that the probability statement is false and is even consistent with the probability statement being true.

Categorical statements are meaningful when the sets involved are finite and meaningless when the sets involved are infinite, whereas probability statements are statements about infinite sets where probability is undefined for finite or empty sets. The former are what we tend to use in classical probability problems, and in these, as in the problem given above of balls in bags, we are dealing with finite sets. Can we make probability statements about these problems? Granted that we cannot estimate the probability prior to drawing a ball from the bag, but can we make sense of estimating the probability even after we have drawn a ball from the bag, given that there are only a finite number of balls in the bag? Isn't probability undefined for this 
scenario?

No; a finesse is possible here. Consider the statement "All the balls in this bag are white". There are twenty balls in the bag, all of them white. As a categorical statement this means that one could draw each ball from the bag without replacing it, state of each that it is white, and when the last ball has been drawn and is stated to be white conclude "All the balls in this bag are white." It should be noted that the categorical statement here merely restates the evidence. This is induction by complete enumeration but, since the conclusion does not go beyond the evidence, this barely deserves the name of induction.

Now, from what I said above it is tempting to think that, because there is a finite number of balls in a bag and probabilistic statements are about infinite sets, one cannot make the probability statement "All the balls in this bag are white". However, the frequency theorist would understand such a statement in the following way: if one were to draw a ball from the bag and then, by replacing the ball, put the bag back into its initial condition, and then repeat this process ad infinitum, then the ratio of white balls drawn to balls drawn would converge on unity. Although the truth of the probability statement is grounded on and made true by facts concerning a finite set (although these facts do not establish it conclusively, as already said), it is not about a finite set (namely, the set of balls) but about an infinite set (namely, the events of possible ball drawings). But prior to drawing a ball, the frequency theorist can make no statement at all about the probability of the hypothesis that all the balls in the bag are white.

This may seem odd. Consider the case where you know that there are only white balls and black balls and that there is only one ball in the bag. It is extremely intuitive, and indeed endorsed by classical probability theory, to say that you are equally likely to draw a white ball as a black ball, and that the probability of "All the balls in the bag are white" is 0.5. But the frequency theorist can't say this, and doesn't. The Straight Rule sometimes described as "more of the same" can only apply when there is some event of which there can be more of; prior to the occurrence of that event it has nothing to say. Before a ball has been drawn the frequency theorist's answer "What is the probability that all the balls in the bag are white?" is "It is impossible to say" (which should not, of course, be confused with saying that the probability is zero). To the question "What is the probability that the next ball I draw will be white" I urge the frequency theorist to give the same answer as classical probability theory, viz., 0.5 .

The grounds of statements about infinite sets is evidence about finite sets, namely, the relative frequency in the sample so far observed - the so-called 'practical limit'. That each ball out of the bag is white (i.e., the frequency series goes $\{1 / 1,2 / 2,3 / 3 \ldots 20 / 20\}$ ) is logically equivalent to the categorical statement "All the balls in the bag are white" but is only the grounds of the probabilistic statement "All 
the balls in the bag are white" and does not establish it conclusively even if the enumeration has been completed; there must still be an ampliative inference from the evidence to the probabilistic statement. In a more typical case we might have a frequency series like $\{1 / 1,2 / 2,3 / 3 \ldots 19 / 20 \ldots\}$ that could in principle continue indefinitely and would be the grounds of our probabilistic statement "Most swans are white (with probability 0.95)." Note that here it is the categorical statement "Most swans are white" that is meaningless because reducible to an infinite set. ${ }^{6}$ The evidence statement would be categorical - a summation of what had already been observed. But the limit in the finite sample we have observed so far may not be the limit in the infinite series; the grounds support the hypothesis only on the assumption that the sample has converged on its final limit. The evidence itself says nothing about those assumptions, so the probability statement presupposes something about the future for which evidence is lacking. Thus, although inductive generalizations have content concerning the future, there are no grounds - inductive or otherwise - for that content; they are part of the assumption that the world is predictable.

\section{Five Debates}

\section{a. Reichenbach and Popper}

I will now show that although choice and belief come together in answering the first question of selecting a hypothesis, the sufficiency of a method for answering it can itself be established only by establishing the rationality of a prior choice. Hence, the question of the rationality of a choice is prior to the question of the rationality of a belief. Both inductivist (taking the justificationism of Reichenbach and Salmon to be representative) and falsificationist (taking Popper to be representative) methods depend on a near-identical claim about choice.

First we have to look at belief, largely because it is with belief, and degrees thereof, that probabilities have usually been associated. It is a psychological fact (generally speaking) that we believe something more strongly the more it is confirmed in our experience and the issue is whether such a belief is justified. Justification has usually been construed on deductivist lines - one is justified in believing the deductive closure of the other things one believes in, logical inferences transmitting justification from premises to conclusion. But deduction only preserves truth and justification; to be justified tout court requires the propositions at the bottom of the inferential chain to be infallibly true and non-inferentially justified, otherwise there is an epistemic regress - the conclusion is justified only on the condition that the premises are justified, and these are justified only on the condition they are the conclusions of arguments whose premises are themselves justified, and so on. In a priori knowledge there are such infallibly true propositions to appeal to so this model of 
justification seems an adequate model for this kind of knowledge, but as a model of empirical knowledge it seems to exclude too much on the grounds that empirical knowledge by its very nature is fallible and those foundational contingent truths from which logical inferences can transmit justification can never be conclusively established.

One natural thought is to think that even if foundations cannot be established infallibly, we can at least establish that they are likely, and these could end the epistemic regress in fallibilistic reasoning just as infallible truth did in deductive reasoning. But this is an illusion, and there is nothing between these forms of justification; in fact, it is easy to show that the same problems arise here as before. Firstly, what has changed here is only the premise, not the way in which justification is propagated. If the premise has a probability then the justification is weaker in the sense that it does not guarantee the truth of the conclusion but it is nonetheless transmitted through deductive relations - these preserve probability in that relative to the premises, ${ }^{7}$ the conclusion is as likely as the premises. But the probability being transmitted here is the epistemic probability and not the empirical probability. Although there are cases when we can 'convert' from empirical probability to epistemic probability, this is only under condition that we are certain of - which is to say, we can conclusively establish - the empirical probability. But this takes us back to square one.

Let me elaborate. Rejecting infallibilism, an inductivist would say that we only need to show the conclusion to be highly probable to be worthy of belief. Now his argument is "The premise is highly probable; therefore, (relative to the premise) the conclusion is highly probable". But the probability that is mentioned in "The premise is highly probable" is the empirical probability contained in the premise (the probability of the hypothesis) and not the epistemic probability; we do not say (although it is not nonsensical to say) that the premise is probably probable. Whatever probability or probability interval is discovered by a rational method and attached to a proposition, that that proposition has that probability is either true or false and this itself is something that has to be established and may be established only with a certain degree of confidence. But this is the problem we started with. The mere involvement of a probability does not in itself lead to a fallibilist model of justification; the probability of p must be established just as conclusively in the latter case as its truth-value is in the first, or, to put it in terms of fallibilism, the latter requires an infallible determination of its value as much as the former.

Does this mean the end for justificationism? Is Popper right to say that it makes no sense to attach probabilities to hypotheses? If he means empirical probabilities, then no; nothing said so far rules out learning the probability of the hypothesis by positive confirmation, by learning from the evidence. But the probability of the hypothesis has now become a technical notion whose relevance to future conduct is 
by no means assured without further argument. If he means the epistemic probability then he may be right, although this does not rule out being able to make a rational choice between hypotheses, as Popper himself allows.

The solution seems to be to reject the geometrical model of knowledge as inapplicable to contingent truths and rethink what we mean by empirical knowledge, and this is basically what Popper and Reichenbach do. Popper says that knowledge is a system of conjectures, Reichenbach that it is a system of posits. There is a basic similarity here; the difference is that Reichenbach still conceives his programme in justificationist terms and Popper conceives his programme in falsificationist terms.

Starting with Popper, I would reconstruct his position as follows. It is irrational to believe a proposition that cannot be established either as true or even as probable. The concept of justification is a normative concept concerning what you should believe: it cannot be the case that you should believe what it is irrational to believe. Therefore, the concept of justification is empty and cannot be integrated into a truly fallibilist model. A fallibilist model must accept that foundational propositions cannot be established and content itself instead with unjustified conjectures, relying on a rational procedure of sincere and severe attempts to falsify the conjecture and over time to impose a preference ordering over the conjectures which, Popper insists, is not a preference for which to believe but for which should be agreed to by the scientific community or otherwise taken as a basis for action, agreement been taken as the surrogate for truth in a fallible world. Having agreed to a particular proposition, Popper would not deny that we should also agree to its logical consequences, but we should not say that the proposition justifies those consequences because there is no justification to be transmitted from a mere conjecture even when it has been agreed to; deriving logical consequences is important because it amounts to the derivation of potential falsifiers and in doing so amounts to a measure of the empirical content of the conjecture. It is rational to agree to or to choose such a proposition even though it cannot be established conclusively either as true or even as probable since, importantly, confirming evidence does not increase the degree of belief, but falsifying evidence tells us what we should not choose, and this purely by deduction. There is no place for induction or for justification or even, it seems, for rational belief.

Reichenbach's position can be put as follows. Knowledge is defined functionally, its function being to guide us in our actions, i.e., to provide us with the best predictions possible. Given the necessity of acting, it is unreasonable to expect the actor to have absolute certainty. We cannot, on the grounds that we can never finally establish a claim that an action will be successful, settle for inaction and hope for the best. It is in this sense that his view can be seen as pragmatic. Thus, given that we are forced to act our best prediction or wager is functionally equivalent ${ }^{8}$ to - i.e., leads to the same actions as - a claim that is demonstrably certain were there to be such, and given that we should believe such a claim, we should likewise believe 
our best wager, at least given a behaviourist analysis of mental states like belief. Thus, Reichenbach rescues the 'belief' in rational belief but only by arguing that it is functionally equivalent to rational choice given the need to act rationally, i.e., on the basis of reliable predictions. His difference from Popper thus appears, in part, a merely verbal point about the meaning-in-use of 'belief'.

Like Popper, Reichenbach concedes that we do not know whether the world is law-like and exhibits regularities, and we cannot even say whether the world's being this way is likely or unlikely. But we can show using only tautologies that the Straight Rule will find those regularities if they do exist, and if they do not exist then we cannot do anything. Therefore, our best wager is to suppose that the world is regular, and then it follows that the Straight Rule answers the question of what hypothesis is most likely and most rational to select, ${ }^{9}$ that is to say, it solves the problem of estimating the probability of the hypothesis.

It should be noted immediately that the concept of a wager is essentially the concept of a choice. For both Popper and Reichenbach, the problem of the truth or probability of foundational propositions is obviated by the practical necessity to choose. When an end has been decided on, a means to that end - a regularity must be presupposed. The difference, perhaps, is that Reichenbach's choice involves a global claim about the world and Popper's choice involves local claims about how he takes that part of the world the hypothesis is about to be. This is more consistent with Popper's indeterminism and his belief that the universe is open, although it is not clear that it is actually inconsistent with Reichenbach's posit of a general uniformity.

It is on these kinds of grounds that the choices underlying both Reichenbach's and Popper's theories are rationalized. When you choose a hypothesis you are not necessarily saying that you believe it or think that it is true, but that having been forced to choose by the necessity to act, Popper argues that the best corroborated theories are the best choice; in practical life, it is often not possible to opt out of playing the game. Because choice of and belief in hypotheses are disconnected for Popper, we certainly should not say that we ought to believe the hypothesis or rely on it in the sense that it justifies our choice. Reichenbach may talk of justification because choice and belief are not for him disconnected but are functionally equivalent. In the end, the difference seems less substantive than at first appears. What is substantive and will be mentioned later is Popper's rejection of positive confirmation and by implication of the Straight Rule as a measure of the rationality of choice.

\section{b. Salmon and Popper}

In some well-known papers Salmon criticizes Popper's falsificationism and Carnap's inductivism as means of making ampliative inferences on the grounds that neither

Principia 18(1): 1-24 (2014). 
corroboration statements nor confirmation statements are ampliative, the former because it is a synthetic statement about the past performance of a hypothesis and says nothing about its future performance, the latter because it is an analytic statement of a priori relations between statements in a (to some degree) arbitrarily defined language. It is with the former critique that this paper is concerned, and will first of all focus on Salmon's claim that corroboration offers no basis for 'practical prediction'.

According to Salmon, the choice of the best corroborated hypothesis is not rational, and it does no good to argue that its alternatives are irrational. I will show that corroboration can be vindicated in much the same way that the Straight Rule can be vindicated and that considerations similar to those that Salmon argues make it rational to choose the best confirmed hypothesis would also make it rational to choose the best corroborated - Salmon's claim to the contrary errs by confusing a semantic issue with an epistemological issue.

Salmon (1981) criticizes Popper on the grounds that corroboration provides no rational basis for practical prediction, but seems not to notice that Popper's answer is much the same as Reichenbach's, which Salmon often endorses. It is difficult to see how Salmon can consistently defend the pragmatic vindication of induction against the objection that it relies on an unjustified claim that the world is law-like and yet criticize falsificationism on the grounds that it does not make selection of the best corroborated hypothesis rational. Both are vindicated on the grounds that once an end is chosen, a means to that end must be presupposed and a hypothesis that has been observed to achieve that end is a rational choice. For Reichenbach, it is rational to choose the Straight Rule and the Straight Rule determines which hypothesis is most likely and most rational to choose. For Popper there does not seem to be this intermediary selection of a rule to further select the hypothesis, but rather the hypothesis is selected directly by virtue of passing critical tests, typically in competition with other hypotheses. In a sense, then, Popper has fewer commitments and it is consistent with his view that there may be parts of the universe that are not regular or whose parameters are beyond those in which the hypothesis can be taken to function reliably.

No doubt Salmon would deny this because he poses the question in terms of whether the prediction - ipso facto a statement about a future event - can be rationalized by statements about past events, and corroboration is a summation of the past performance of a hypothesis, namely that it has so far withstood critical tests, and explicitly said by Popper not to be a guide to future performance. In contrast, an inductive generalization such as delivered by the Straight Rule is a statement about the future as well as the past; without induction, Salmon (1981) concludes, no prediction or truth-valuable statement about the future is rational. But I would argue against Salmon that Reichenbach does not provide that the evidence so far is evidence also that the future will resemble the past - that it will be is simply 
posited. What we are justified in saying is that if this series converges on a value, this value (the practical limit) is our best wager of what that value is. That the series is, in fact, convergent, is part of the supposition that the world is regular. I am not saying that Salmon is unaware of this, but that he makes a mistake by concerning himself only with the meaning of the inductive generalization without considering what parts of the generalization are justified and what part is merely supposed; he makes a semantic point when he needs to make an epistemological point. Perhaps Salmon would point out that a prediction can be inferred from an inductive generalization whereas it cannot be from a statement only about the past. But I don't think this helps, since it would be inferred only relative to a supposition, i.e., a proposition that is not, as Salmon would agree, justified by the evidence.

Reichenbach's pragmatic justification of induction then has much in common with Popper's defence of corroboration, and they both stand or fall depending on whether the choice involved is rational. In the long run, the Straight Rule will find whatever regularities exist and falsification will eliminate all hypotheses except the true ones supposing there to be any true ones. For the choice to be rational it must, I think, be shown that the alternative choices are irrational or at the very least not more rational. Thus, one of the problems with Reichenbach's pragmatic justification of induction is that what it shows is only that the rule chosen must be asymptotic and does not pick out the Straight Rule (or any other asymptotic rule) as the most rational.

This goes contrary in spirit to Miller (n.d.) who, defending the idea that the choice itself, rather than the hypothesis on which it is based, can be subjected to critical tests and should be adopted if it survives them, adopts a satisficing concept of rationality where the issue is not why the choice made is rational but why the alternative choices are irrational. This seems evasive; it seems analogous to the claim that it is unnecessary to find a reason to prefer one asymptotic rule over another because they all converge eventually on the same limit. But this makes inference to the next instance irrational because there is an asymptotic rule that will give any value that one may arbitrarily choose for the next instance.

Being fair to Miller, he does provide an argument to the effect that a proposal that has passed critical tests should be preferred to another that, although empirically adequate, has not been critically tested. Although he does not mention this, his argument seems to resemble that of experimental realism: proposals make use of low-level generalizations rather than hypotheses (which, Miller seems to concede, are vulnerable to Salmon's critique of corroboration), and once shown to satisfice the end for which it was chosen (or as experimental realists put it, used in the design of a functioning machine) there is no requirement to critically test the alternatives. We are rational in adopting the tested proposal in an analogous way to which we are rational in believing in the existence of those entities whose causal powers (as 
represented by low-level generalizations) we exploited in the design of the machine. Choosing the untested proposal, says Miller (n.d., p.15-16) constitutes

a grave dereliction of rational duty, an infraction that cannot be excused by the plea that, had the examination taken place, it would have had no effect on the decision ... This does not mean that the decision to adopt [the alternative] is more likely to be disastrous. The repudiation ... is entirely methodological, and is independent of whether it might be a success ... $[\mathrm{T}]$ he subject is the rational making of decisions, not the making of rational decisions.

Ingenious as all this is, it is not clear to me how this advances on the original suggestion that the best corroborated hypothesis be used for the purposes of practical prediction. It is not clear to me what the move from hypotheses to low-level generalizations is meant to achieve. As an anti-inductivist, Miller insists (n.d., p.8-9) that the low-level generalizations are not established by induction (or by complete enumeration, distinguishing this for a moment from induction). But if they are established by corroboration, then Miller is relying on the fact that it is more rational to test proposals using better corroborated low-level generalizations than less well corroborated low-level generalizations. But if Salmon's critique is correct (and Miller seems to concede that it is) then it applies equally here. ${ }^{10}$ In addition, I think that Miller's account gains plausibility from its "all or nothing" evaluation of the proposal. There will be occasions where the proposal does not achieve its end, just as any factory line has its fair share of rejects. Then it is obviously relevant which proposal works better in terms of leading to fewer errors and it would be irrational to choose the proposal that leads to more errors even if that proposal satisfices. Miller's conception seems to have more in common with a deductive argument where it is no more rational to choose a short proof over a lengthy proof or, as I mentioned before, one asymptotic rule over another if all were to be evaluated "in the long run". But this does not apply when the next instance or next output in the factory line is considered. If I need to deliver 100 burger-boxes to McDonald's I am better off choosing a process where the proportion of rejects is lower rather than higher. A satisficing conception of rationality is, well, unsatisfactory.

Where Reichenbach, I think, improves on Popper's position is that he notices that posits can be appraised - that is to say, given probabilities - relative to higher-level or so-called 'blind' posits irrespective of the fact that blind posits do not have probabilities. This allows him to continue to talk about beliefs being justified on the grounds of being more likely, which is to say, better confirmed by positive instances. Rational choice of a hypothesis and rational belief in a hypothesis is a distinction without a difference when the purpose for which the choice is made is simply to approximate the truth, i.e., the theoretical explication of the universe. Similarly for 
falsificationism, Salmon concedes for the sake of argument Popper's claim that corroboration is a rational basis for 'theoretical prediction'. If you need to achieve empirical knowledge, and empirical knowledge is only achievable if the world is law-like, then it is rational to act as if the world is law-like. The rationality of the belief is dependent on the rationality of this choice. If this choice is rational for Reichenbach then it is rational for Popper. And it is rational.

\section{c. Popper and Carnap}

In this section I will discuss several arguments by Popper and Carnap and argue that they founder either because of a mistaken interpretation of the probability statement or because of being misdirected at a different problem.

Popper attempts to show that no number of positive instances can affect the probability of a hypothesis. If he is right then it is irrational to choose the best confirmed hypothesis. I have argued that the rationality of choosing the best confirmed hypothesis and the best corroborated hypothesis are in much the same boat. Thus, my purpose must be to show that Popper's argument goes wrong and does not leave corroboration as sole occupier of the field.

Return to the example we began with. Consider now the situation where we draw balls from only one of the bags but we don't know whether this bag is the one with the black ball. It is tempting to think now that the probability of the twentieth being black is still 0.5, quite irrespective of the evidence of previous drawings. Popper (1962) argues as follows (I have adapted his example slightly to match the above): suppose that all the balls except one have been drawn and have all been found to be white. Take $a$ as the name of the statement "All the balls in our selected bag are white" and $b$ as the name of the statement "Precisely one ball in our selected bag is black". What is the probability of the statement $a$, given the information $a$ or $b$, or equivalently, what is the probability of all balls being white, given that either all are white or exactly one is black? The formula for this is

$$
p(a, a \text { or } b)=p(a) /(p(a)+p(b)-p(a b))
$$

Since $a$ and $b$ are incompatible their intersection must be null, i.e., $p(a b)=0$. This gives $p(a, a$ or $b)=0.5 /(0.5+0.5)=0.5 / 1.0=0.5$. In other words, only the weight of evidence, decided a prioristically, is left, the probability of the hypothesis and consequently the evidence for it being rendered irrelevant.

The conclusions Popper (1962, p.71) draws are dramatic:

Our formula does not refer to [the size of the sample]. It is, therefore, valid for [samples] of all sizes (and it may even be extended to infinite samples). It shows that, even on the assumption that we have checked all [members]

Principia 18(1): 1-24 (2014). 
of a sequence (even an infinite sequence) except, say, the first, and found that they all have the property A, this information does not raise the probability that the as yet unknown first [member] has the property A rather than B.

Thus the probability of a universal law — such as the statement $a-$ remains, on our assumptions ... equal to $f=1 / 2$ even if the number of supporting observations becomes infinite, provided one case of probability $r=1 / 2$ remains unobserved.

This method of working out the probabilities amounts, in Carnap's $\lambda$-system, to $\lambda=\infty$ or in other words giving weight to the 'logical factor' (where a Principle of Indifference determines that all logical possibilities — or in Carnap's modified version, all structure-descriptions - are given equal probabilities) and zero weight to the 'empirical factor'. Carnap (1952, p.38) rejects this method because it has as a consequence (here apparently embraced by Popper) that we do not learn anything from experience and he uses instead the rule $P=\left(s_{A}+1\right) /(s+2)$ where $s$ is the number of balls in a sample and $s_{A}$ is the number of these that are white. If we have examined every ball in the population apart from one (i.e., $s_{A}=n-1=20-1=19$ ) then this reduces to $P=n /(n+1)=20 / 21$ (Popper 1962, p.72). Popper seems to be questioning the rationality of the choice of one rule rather than another from the $\lambda$-system and seems sceptical about arguing abductively, so to speak, from the rule's effects. His overall intention seems to be to show that there never could be a rational choice, and this is to be expected because it makes no sense to attach probabilities to hypotheses in the first place.

One curious thing about Popper's paper is that although he takes himself to be talking about singular predictive inferences (Popper 1962, p.69, 71) $a$ is not a singular statement at all but a general one, and in his conclusion is said to be a universal law. He seems to take this to be equivalent to a singular statement in the case where every member of the population except one has been observed. Since observing the only as yet unobserved member will complete the enumeration he takes the probability of its being A as the probability that they are all A (Popper 1962, p.70). But this is wrong because it takes $a$ not as a probability statement but as a categorical one.

The question "What is the probability of all balls being white, given that either all are white or exactly one is black?" read probabilistically means "What is the frequency with which I would expect to draw white balls if I were to draw a ball, replace it, and repeat this ad infinitum, given that all the balls drawn so far have been white?" And this is the question we have already answered, and the same answer is given whether in the actual trial there is only one ball left in the bag or still twenty balls. The fact that I may have drawn from a different bag means only that if I am asked to bet on a being true then I might not do so, since prior to 
any drawings the logical factor does not favour the choice of one bag over another. However, this logical factor decreases as the sample size increases since the weight of evidence that the bag being drawn from is the one with the black ball decreases as one draws white balls, since if one had been drawing from the bag that had the black ball then the probability that one would have drawn it by now increases as the number of drawings increases, therefore the fact that one has not drawn the black ball is evidence against it being the bag with the black ball and I become less willing to bet on this hypothesis. In other words, the information "or there is precisely one black ball" does not change the probability of the hypothesis which depends only on the evidence given in the frequency series but does change our certainty over the truth of that hypothesis and the rational betting quotient for the next instance. The correct answer for the probability of the next ball drawn being black using empirical probabilities should be slightly less than 0.5 because the balls so far drawn all being white is slightly more probable given the hypothesis that the bag drawn from has twenty white balls than it is given the hypothesis that the bag drawn from has nineteen white balls and one black ball. And this seems correct since Popper, of all people, cannot assume that because a ball was black before it is still black now.

In this way our practical prediction and our beliefs can come apart. We are not at all certain that the probability is 1.0, but this does not mean that we believe that the probability is other than 1.0 or that this is not the answer we would give for the probability of the hypothesis if we were forced to give one. For suppose now that we are asked to bet that a hypothesis is true. Having drawn the white ball, we do not bet that the proportion of white balls to black balls in the bag is 0.975 . Indeed, we know that this hypothesis is false because it would amount to something like one ball being half-black and half-white! In the long term the probability will converge on whatever the ratio of white balls to black balls is, but while only white balls have been drawn, then knowing that drawing a white ball is more likely given twenty balls being white than nineteen being white, we bet, if we bet at all, on the hypothesis that all the balls in the bag are white. And this, it should be noted, is what the Straight Rule would give as the practical limit and empirical factor. The difference made by the presence of the additional bag is that we will require greater inducement to be forced to bet at all.

This goes also for the problem of 'variety of instances'. Limited variety may make us not at all certain that the sample from which we are making our inference is representative, but this does not affect the probability of the hypothesis itself but only our confidence in it as a basis of action. Therefore, the choice is rational and it is still rational even if you do not believe the chosen hypothesis to be true and even think it unlikely, provided that you think it is better than the alternatives and it is worthwhile, given the potential costs and rewards of playing, to play the game. This 
seems to me what Popper has actually shown by his argument, and not anything about singular predictive inferences.

So, we should beware of confusing the probability of the hypothesis and the weight of evidence. The probability of the hypothesis is always given (although I concede that I cannot demonstrate this) by the Straight Rule. Carnap's preferred rule referred to in Popper's example, being different from the Straight Rule, is, I think, wrong. However, Carnap is right in so far as a satisfactory answer to the question of the probability of the next instance requires some rule involving both a logical and an empirical factor. Consider the case where you know that there are both white balls and black balls but have not yet drawn one, and consider the question "What is the probability that the first ball that you draw will be white?" As already said, the traditional frequency theorist must reject the question because he recognizes only an empirical factor, but if we take a logical factor into account then we would get 0.5 as we would intuitively expect. Then you begin to amass evidence by drawing balls. But let us suppose that you believe the sample you have taken so far not to be representative. Then, although as the sample size increases (and the probability that it is biased decreases) more and more weight will be put onto the empirical factor and less and less weight will be put on the logical factor, you will not necessarily eliminate the logical factor completely until it is believed that the frequency series has converged. So, for instance, the probability that the next ball that you draw will be white is something like ((the probability that the sample is unbiased $\mathrm{x}$ the limit in that sample) + (the probability that the sample is biased / number of possible outcomes for the next ball)).

In short, Carnap's $\lambda$-system (which is an account of singular predictive inferences) seems correct in its conception, at least, and its critique of the Straight Rule is justified were the Straight Rule to be used for singular predictive inferences. But the critique does not show the Straight Rule to be the wrong method for determining the probability of the hypothesis itself. What is this critique?

\section{d. Carnap and Reichenbach}

In this section I will argue that Carnap's critique supports my contention that the Straight Rule is in general inadequate for solving the problem of singular predictive inferences but does not support Carnap's claim that the Straight Rule is to be rejected outright.

Carnap criticizes the straight rule $(\lambda=0)$ for similar reasons for which he criticized the rule $\lambda=\infty$, namely because you do not seem to learn from it. The Straight Rule says "Always take the practical limit" as the limit of the infinite series irrespective of the sample size. The practical limit after drawing two white balls from the bag is 1.0, and the practical limit after drawing twenty white balls from the bag is 
1.0. This also suggests that we would be prepared to accept exactly the same betting odds after two drawings as after twenty (Carnap 1952, p.42-43). We do not seem to have learnt anything after the twentieth drawing that we didn't already know after the second or the first.

This is basically the paradox of ideal evidence. Positive evidence, we would normally say, should raise the probability of the hypothesis. But here the probability of the hypothesis is set at 1.0 from the start and never moves. The usual response in the case of ideal evidence is basically the same as what I have called interchangeably willingness to bet, weight of evidence, or epistemic probability - what increases with sample size is willingness to bet or, what is really the same for the inductivist, the probability that the sample is representative. ${ }^{11}$

Similarly Carnap (1952, p.42) gives the following scenario as leading the Straight Rule into counter-intuitive results. Suppose that we have observed instances of $P_{1}$ and instances of $P_{2}$. These are such that their conjunction is logically possible but have never yet been observed. The Straight Rule says that the probability of something's being both $P_{1}$ and $P_{2}$ is 0.0 because it has never been observed, but Carnap objects that something that is logically compatible with the evidence cannot have a probability of 0.0 . My response is as before. We should not infer that the probability of the next instance is 0.0 , but there is nothing counter-intuitive in inferring that the probability of the hypothesis is 0.0 .

I will put Carnap's (1952, p.43) next scenario in his own words:

If all we know about the universe is that the only observed thing is $\mathrm{M}$, then the method of the straight rule leads us to assume with inductive certainty all things are $\mathrm{M}$ and to take the estimate of the $\mathrm{rf}$ [relative frequency] of $\mathrm{M}$ in the universe to be $1 \ldots$ Thus, on the basis of the observation of just one thing, which was found to be a black dog, the method of the straight rule declares a betting quotient of 1 to be fair for a bet on the prediction that the next thing will again be a black dog and likewise for a bet on the prediction that all things without a single exception will be a black dog ... Thus, this method tells you that that if you bet ... on either of the two predictions mentioned, while the stake of your partner is 0 , then this is a fair bet.

Carnap concludes that this is obviously not a fair bet, and any method that tells you it is must therefore be wrong. Interestingly, this seems to reduce to the previous scenario, for presuming that being a black dog and being observable are logically independent, it is logically possible for there to be unobserved black dogs and observed (at some point) things that are not black dogs. Perhaps Carnap would reject this because, as Popper (1962) notes, observability is not a predicate in Carnap's ideal language.

Although distinguishing the two ends of inductive methods and focussing on the singular predictive inference, Carnap seems to slip back to the other question by fail- 
ing to notice that betting on a hypothesis being true is equivalent to the first of those ends and not really a singular predictive inference. As Salmon remarks, it does not really make sense to lay bets about the truth of probability statements since these concern infinite sets and hence can never be settled one way or the other. It only makes sense to bet on the next instance or a finite number of unobserved instances, i.e., something completely enumerable and hence expressible by a categorical statement, and the probability of this, I have argued, is not given purely by the Straight Rule. Carnap is right to say that the Straight Rule is inadequate for this, but it does not follow, as he seems to say that it does, that the Straight Rule is inadequate for determining the empirical factor; he does not keep to his own programme.

\section{e. van Fraassen and Lipton}

Defenders of inference to the best explanation like Lipton argue that explanatory considerations should be able to affect the probability of the hypothesis. Opposing this view, van Fraassen argues via a Dutch book argument that if we allow this we are can end up with beliefs that are incoherent.

The literature is too voluminous to be discussed here. My basic strategy should by now be familiar. Assuming van Fraassen to be correct with regard to the probability of the hypothesis, this does not rule out the use of explanatory factors for the problem of the singular predictive inference. Plausibly, explanatory virtue affects the logical factor; it is not forced upon us that we give every alternative hypothesis equal probability when trying to infer to the next instance. Assume that you know that there are white balls in existence but not that there are black balls. The hypothesis that "All the balls in the bag are white" is simpler than "Most of the balls in the bag are white but one is black" because it has fewer ontological commitments, namely it is not committed to black balls. This doesn't mean that one should, for that reason, believe that "All the balls in the bag are white"; explanatory virtue does not alter the probability of the hypothesis. But it does, I suggest, alter the willingness to bet or weight of evidence; for instance, in the two-bag scenario described earlier, instead of 0.5 the weight of evidence given to the hypothesis that "All the balls in the bag are white" might be upgraded to 0.6 , giving us $((0.6 * 1.0)+(0.4 * 0.95))=0.98$ rather than 0.975 as the probability that the ball you draw next will be white.

Similarly, Harman's (1965) argument that the validity of the inference from the sample to the population depends on explanatory considerations — in particular whether the observed regularity is better explained as due to a genuine law-like regularity than by the sample being biased - does not show as he claims that induction collapses into inference to the best explanation or that we should not make generalizations unless sufficiently confident in a prior judgment that the sample is unbiased. As applied against inductive generalization his objections are unsound, but the gen-

Principia 18(1): 1-24 (2014). 
eral points he makes can plausibly be taken as showing that such considerations can enter into inference to the next instance. There must be a logical and an empirical factor, as already said.

I am inclined to reject inference to the best explanation as giving additional reasons to believe hypotheses because I believe it to be at best redundant, being no different in the end from hypothetico-deductivism, and at worst it is possibly incoherent as van Fraassen argues. The probability of a hypothesis depends only on its positive instances irrespective of their variety or of how well they are explained.

\section{Conclusion}

Empirical evidence does not in itself tell us what hypothesis is true or what outcomes to expect. Evidence has weight only conditional on other substantive assumptions about the world. Our belief that these facts are true cannot be justified either deductively or inductively, but a pragmatic attitude towards them can be warranted on the grounds that it results from a rational choice. This choice is rational in the instrumental sense that it will produce knowledge if anything will. Having chosen an end, we must perforce be prepared to take there to be a means to that end. Justificationism and falsificationism alike require such rational choices to get started. Although I have favoured justificationism I have not shown that falsificationism is a mistaken project or that corroboration could not be a rational basis for both theoretical and practical prediction. My preference for justificationism is based on the fact that since whatever reasons we might appeal to in its favour are the same also in the case of falsificationism, and justificationism seems more powerful and based on principles of the probability calculus that are well understood, it seems good policy to favour justificationism. Also, it means that our psychological inclination to believe more strongly what is better confirmed by positive evidence is not an epistemological mistake.

Generally, we are rational to believe the hypotheses that we do believe. But we are not always rational in betting that the next instance will be what the rationally believed hypothesis predicts it will be. This is a different kind of problem requiring consideration of more factors. This is suggested both by the ideas behind Carnap's $\lambda$-system and the intuitiveness of inference to the best explanation; perhaps explanatory considerations will turn out to have an effect on our choice of $\lambda$. I don't claim to know how this will work itself out, but future expositions of inference to the best explanation should be framed within a discussion of the singular predictive inference and is perhaps the same as or a sub-type of the singular predictive inference. ${ }^{12}$

Principia 18(1): 1-24 (2014). 


\section{References}

Carnap, R. 1952. The continuum of inductive methods. Chicago: University of Chicago Press. Engel, P. (ed.). 2000. Believing and accepting. Dordrecht, The Netherlands: Kluwer Academic Publishers.

Harman, G. 1965. Inference to the best explanation. The Philosophical Review 74(1): 88-95. Hintikka, J. 1965. Replies to comments on Hintikka's "Induction by enumeration and elimination". In: I. Lakatos (ed.) The problem of inductive logic. Amsterdam: North-Holland Publishing Company.

Miller, D. n.d. Deductivist decision making. Available at: www2.warwick.ac.uk/fac/soc/philosophy/people/associates/miller/kpf.pdf. Last accessed 9th March 2011

Mura, A. 1998. Hume's inductive logic. Synthese 113: 303-331.

Popper, K. 1962. On Carnap's version of Laplace's rule of succession. Mind 71: 69-73.

Reichenbach, H. 1968. Experience and prediction. Phoenix Books. Chicago: The University of Chicago Press.

Salmon, W. 1981. Rational prediction. The British Journal for the Philosophy of Science 32(2): 115-25.

DAVID BotTING

Universidade Nova de Lisboa

IFL, FCSH

Lisbon, Portugal

davidbotting33@yahoo.co.uk

Resumo. Neste artigo discutirei a racionalidade do raciocínio sobre o futuro. Há duas coisas que gostaríamos de saber acerca do futuro: quais hipóteses são verdadeiras e o que vai acontecer em seguida. Para colocar em linguagem filosófica, busco mostrar que existem métodos pelos quais inferir para uma generalização (selecionar uma hipótese) e inferir para a próxima instância (inferência preditiva singular) podem ser mostrados serem normativos e o próprio método pode ser mostrado como sendo racional, onde isso é devido em parte a ele ser baseado em evidência (apesar de não o ser no mesmo sentido) e em parte em uma escolha racional prévia. Também argumentarei que estas duas inferências tem sido confundidas, sendo distintas não apenas conceitualmente (como ninguém duvida) mas também em seus resultados (o valor dado para a probabilidade da hipótese não sendo em geral aquele dado para a próxima instância) e que métodos que são adequados para uma não são por si só adequados para a outra. Um número de debates funda-se sobre esta confusão e não mostra o que seus debatedores pensam que mostram.

Palavras-chave: Probabilidade; escolha racional; confirmação de hipóteses; inferência preditiva singular; indutivismo; falseacionismo.

\section{Notes}

${ }^{1}$ This practical attitude is probably what is referred to by Popper's term "practical belief", van Fraassen's "acceptance", and is perhaps that which should be concluded from Peirce's

Principia 18(1): 1-24 (2014). 
abductive arguments. See Engel (2000) for a discussion of the concept of acceptance and its differences from belief.

${ }^{2}$ Indeed, the statement "The probability that the next ball that I draw will be white is p" is often taken as just a disguised restatement of the hypothesis or of the evidence.

${ }^{3}$ In the future I will speak only of probability and leave degree of corroboration as the implicit alternative. Degrees of corroboration are expressed, like probabilities, as real numbers between 0 and 1 , but do not obey the axioms of probability theory.

Of course, the thesis brackets considerations concerning favourable offers of odds and different utilities of outcomes; these alter what it is rational to choose such that it is not necessarily rational to bet on the next ball being white even if this probability is high. The thesis is rather that the probability that gets fed into whatever decision-theoretical calculation needs to be made is not the probability of the hypothesis but is nevertheless a probability based only on the evidence. When this probability is high I deem the choice rational.

${ }^{4}$ I am here disagreeing with Hintikka's (1965) index of caution. Hintikka argues that, whereas Carnap uses $\lambda$ as an a priori factor in the context of singular predictive inferences, there is similarly $\alpha$ as an a priori factor - an index of caution - for making generalizations. In contrast, I claim that generalizations should always have the values given to them by the frequency series (if we are using the Straight Rule then this is the value of the 'practical limit') and that the problem is not with making generalizations but in basing actions on them, or in other words, betting on the next instance, for which I have agreed that other factors are involved. See Mura (1998) for a fuller explanation of the concept weight of evidence.

${ }^{5}$ The logical notation I use for this kind of statement, namely $\forall x . X(x) \supset^{1.0} Y(x)$, is informative in one way but misleading in another. It is informative in that the probability is attached to the connective and not as a modal operator to the consequent; it does not mean that $\mathrm{Y}(\mathrm{x})$ has a probability instead of a truth-value. It means instead "Being an X makes it probable with a probability of 1.0 of whatever it is that is an X that it is a Y." It is misleading because I have persisted in using a universal quantifier which makes it looks as if it were a general rather than a singular statement, and also might be taken to imply that, like the ordinary $\forall x . X(x) \supset Y(x)$, it could be vacuously true; whereas the categorical "All Xs are Y" is true if there are no Xs, the probabilistic "All Xs are $Y$ " is meaningless because the probability is undefined if the sets are not infinite, which they are not if there are no Xs or no Ys, i.e., the sets are empty. An intensional construal of the quantifier must be insisted upon exclusively. As an example of what I mean by an intensional construal it can be seen that in a categorical statement the quantifier can also be considered intensionally, when it becomes a singular statement concerning the properties of the classes $\mathrm{X}$ and $\mathrm{Y}$ whose extensions may be infinite, finite, or empty. Thus, when the classes are infinite an intensional reading is still meaningful whereas the extensional reading is not because unverifiable. There is, then, similarity between the intensional categorical statement and the probability statement, but they are not the same because the probability statement is consistent with the existence of negative instances and is inconsistent with the non-existence of any instances at all of its reference class, whereas this is not the case for the intensional categorical statement.

${ }^{6}$ This is my way of dealing with the problem rather than Reichenbach's, who would deny that there is a reduction to an infinite set but a probabilistic relation to an infinite set. An empirical generalization like "Most swans are white" would be meaningful because there is such a probabilistic relation. It is reducibility to/logical equivalence to an infinite set that

Principia 18(1): 1-24 (2014). 
would result in meaninglessness. The same technique would also work for "All swans are white" and even for a singular statements like "I see a swan" that is not reduced to a set of protocol statements but is related to those protocol statements probabilistically. Because a probabilistic relation allows an inference about an infinite set to be made on the basis of observed frequencies in a finite set, infinitude does not result in meaninglessness on this theory of meaning.

${ }^{7}$ The qualification is important because what is likely relative to one set of premises may be unlikely relative to another. This does not alter the relationship between the premises and the conclusion, which remains deductive, but does mean that we should not 'detach' the conclusion. Detachment may be appropriate in conditions of infallibility but not elsewhere. In my view detachability is best thought of as an epistemic property of a proposition rather than as a semantic property or a rule.

${ }^{8}$ According to "the principle of the connection of meaning and action" that can be operationalized as "If two sentences will lead us under all possible conditions to the same actions, they have the same meaning" (Reichenbach 1938, p.80) this functional equivalence is also a meaning-equivalence (the "meaning" referred to here seems to be what Reichenbach calls "physical probability meaning"). It follows, although he does not say this explicitly, that "p is true" and "p is our best wager" mean the same thing. I do not intend to go into the bells and whistles Reichenbach adds to the Verification Principle but the following illustrations are interesting because they seem to show that sentences containing super-empirical terms (of which truth, I think, might be considered) mean the same thing as sentences with empirical terms. An actor, it could be objected, could act on such super-empirical beliefs, e.g., refuse to kill cats because of a belief in the divinity of cats. Does this make the functional definition of knowledge too permissive and bring back those metaphysical entities the positivists were so keen to reject as meaningless? Reichenbach says no. The super-empirical meaning can be coordinated with some empirical fact by which the actor decides whether a particular observable, like a cat, satisfies the super-empirical description. For example, the divinity of cats is coordinated to some property of cats that inspires awe in its worshippers. Every action that follows upon the belief that a particular cat is divine follows also on the belief that the cat has the coordinated empirical property (Reichenbach 1938, p.64-67). I am content to omit this talk of meaning and content myself with functional equivalence.

${ }^{9}$ I leave to one side for the moment that the problem that the Straight Rule is not the only such answer and that analogous argumentation supports any asymptotic rule.

${ }^{10}$ Perhaps Miller's thought is that low-level generalizations, unlike hypotheses, allow for exceptions and thus do not claim to be strictly true but are, in fact, known to be false outside of normal operating parameters.

11 There is nothing special about the value 1.0. Suppose that one tossed a coin 1000 times and tossed a head 500 times. Obviously the probability of heads being tossed is 0.5 . Then we toss it a further 1000 times and toss a head 500 times again. The probability is still 0.5. So what have we learned from the additional evidence? Obviously not that tossing a head is any more or less likely. What does seem more likely is that the coin being tossed is a fair one.

Because in the case of ideal evidence the evidence affects the epistemic probability, it should not be inferred that it is the only thing that affects epistemic probability, even in these special cases. I may - because I have not tossed the coin under enough different circumstances, for 
instance - not be very confident that the observed limit is the limit in the population. But it would not make sense to conclude that the probability of the hypothesis should be lowered, rather my degree of confidence that it is true is lowered. Similarly the more representative I believe my sample to be, the more confident I am. This tells against Harman's claim that inductive generalization collapses into inference to the best explanation because dependent upon a prior judgment that the probability we get is better explained as due to a genuine regularity than by the sample being biased (Harman 1965). As a point against inductive inference from sample to population it is false — we do not need any such judgment — but as a point against inductive inference from sample to the next instance it is true. Such a judgment will affect the logical factor. We will return to this in the fifth debate.

12 The author would like to acknowledge financial support from the Portuguese Foundation for Science and Technology (FCT) from grants "Is moral reasoning essentially dialogical?" (SFRH/BPD/77687/2011) and "Argumentation, Communication and Context"(PTDC/FILFIL/110117/2009)

Principia 18(1): 1-24 (2014). 